\section{Hydrogen-bonding Patterns and Ice Nucleation}

INVESTIGATIONS of hydrogen-bonding patterns of organic crystal surfaces in these laboratories have shown that the ability to nucleate ice can be predicted reliably if the complete crystallographic structure is known. Both 'parametric' fit and the density of hydrogen. bonding groups are essential factors conferring nucleative ability, and it is on theso principles (which will form the subject of a more detailed presentation in the near future) that a new organic highly effective nucleating material has been forecast for the first time.

A consideration of the crystal structurel of the $\alpha$ isomer of $1: 3: 5$-cyclohexanetriol dihydrate ( $\alpha$-phloroglucitol dihydrate) led us to expect high nucleative power, which was confirmed by cloud chamber tests. Counts of up to $10^{11}$ nuclei/g were obtained at a temperature of $-12^{\circ} \mathrm{C}$, although owing to the high solubility of the compound tests could not be run above $-8^{\circ} \mathrm{C}$. In comparison, the most active organic nucleators known at the present time (phloroglucinol dihydrate and $\alpha$-phenazine) produce $10^{11}-10^{12}$ nuclei/g under identical conditions, whereas silver iodide is slightly more active still.

Each oxygen atom in the 111 plane of the rhombohedral $\alpha$-phloroglucitol dihydrate corresponds to an ice oxygen, the disregistry being 4.8 per cent. The density of oxygen groups is very close to that of ice and about 50 per cent greater than on the 100 plane of phloroglucinol dihydrate. $\alpha$-Phloroglucitol, being a polyalcohol, shows a much higher solubility in water and it is also more difficult to sublime without decomposition. It does not, however, possess a sweet taste like phloroglucinol as stated in Beilstein.

The compound was prepared by the method of Wislicenus ${ }^{2}$ in which phloroglucinol is hydrogenated with sodium amalgam. After removing all traces of phloroglucinol as well as the $\beta$-isomer of phloroglucitol, the correct m.p. of $184^{\circ} \mathrm{C}$ was obtained.

Because of its alicyclic nature and polyalcoholic character, $\alpha$-phloroglucitol is probably non-toxic.

$$
\text { V. A. Garten }
$$

R. B. HEAD

C.S.I.R.O. Division of Physical Chemistry,

Chemical Research Laboratories, Melbourne.

'Anderson, P., and Hassel, O., Acta Chem. Scand., 2, 5, 537 (1948).

2Wislicenas, W., Ber., 27, 357 (1894).

\section{High-pressure Polymorph of Zirconium Pyrophosphate}

ZirconIUM pyrophosphate is one of a group of isostructural compounds of the Type $X^{+4} \quad \mathrm{P}_{2} \mathrm{O}_{7}$ where $X$ may be zirconium, hafnium, titanium, silicon, tin or uranium ${ }^{1,2}$. It is cubic (Type $K 6_{1}$ ) with zirconium octahedrally, and phosphorus tetrahedrally, co-ordinated with oxygen. The structure may be compared with that of $\mathrm{NaCl}$ (Type $\mathrm{Bl}$ ) by considering the zirconium ions and $\mathrm{P}_{2} \mathrm{O}_{7}$ groups to be at sodium and chlorine sites, respectively.

Reagent grade $\mathrm{ZrP}_{2} \mathrm{O}_{7}$ containing $\sim 12$ per cent of water, which corresponds to $\mathrm{ZrP}_{2} \mathrm{O}_{7} \cdot 2 \mathrm{H}_{2} \mathrm{O}$, was obtained from Bios Laboratories, Ino., New York. This material was heated in air at $700^{\circ} \mathrm{C}$ for $4 \mathrm{~h}$, and its identity as anhydrous cubic $\mathrm{ZrP}_{2} \mathrm{O}_{7}$ was confirmed by $\mathrm{X}$-ray powder diffraction $\left(a_{0}=8.26 \AA\right.$ in agreement with the observation by Harrison et al. ${ }^{3}$ ). The anhydrous $\mathrm{ZrP}_{2} \mathrm{O}_{7}$ was subjected to pressures between 25 and 100 kbars and temperatures of $750^{\circ}$ and $1,000^{\circ} \mathrm{C}$ in a 'belt', samples were quenched under pressure and then examined optically and by X-ray powder methods. The conditions and results of these experiments are given in Table 1.

One hundred per cent conversion to a new quenchable high-pressure polymorph of $\mathrm{ZrP}_{2} \mathrm{O}_{7}$ was effected at 55 and 100 kbars. The new phase is colourless under the microscope and white in powder form. It is uniaxial positive
Table 1. HIGH-PRESSURE HI(HH-TEMPERATLRE FXPERIMENTS ON $\mathrm{Z} / \mathrm{rP}_{2}\left(\mathrm{O}_{2}\right.$

\begin{tabular}{cccl}
$\begin{array}{c}\text { Pressure } \\
\text { (kbars) }\end{array}$ & $\begin{array}{c}\text { Temperature } \\
\left({ }^{\circ} \mathrm{C}\right)\end{array}$ & $\begin{array}{c}\text { Time } \\
(\mathrm{h})\end{array}$ & \multicolumn{1}{c}{ Product } \\
25 & 1,000 & 18 & Cubic $\mathrm{ZrP}_{\mathbf{2}} \mathrm{O}_{7}$ \\
35 & 750 & 19 & Cubic $\mathrm{ZrP}_{2} \mathrm{O}_{7}$ \\
55 & 750 & 20 & High-pressure polymorph \\
100 & 1,000 & 3 & High-pressure polymorph
\end{tabular}

The 100-kbar run was made in a 'girdle' apparatus; the others were made in a 'belt' apparatus

Table 2. X-RAY POWDER DIFFraction Data For High-Pressure Poly(Mn-filtered FeK $a_{1}$ radiation, $\lambda=1.93597 \AA, 57 \cdot 3 \mathrm{~mm}$ camera)

$d(\AA)$
4.76
3.72
3.465
3.316
3.207
3.155
2.948
2.597
2.445
2.411
2.319
2.160
2.041
1.873
1.806
1.748
1.632
1.615
1.586
1.521
1.485
1.462
1.449
1.420
1.392
1.382
1.353
1.328

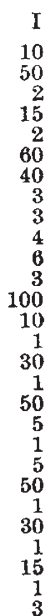

$d(\AA)$

$\begin{array}{rl}\mathrm{I} & d(\AA) \\ 10 & 1 \cdot 302 \\ 50 & 1.281 \\ 2 & 1 \cdot 272 \\ 15 & 1.253 \\ 2 & 1.227 \\ 60 & 1 \cdot 214 \\ 40 & 1 \cdot 167 \\ 3 & 1 \cdot 156 \\ 3 & 1 \cdot 135 \\ 4 & 1.115 \\ 6 & 1.099 \\ 3 & 1.087 \\ 100 & 1 \cdot 074 B \\ 10 & 1.061 \\ 1 & 1 \cdot 055 \\ 30 & 1.054 \\ 1 & 1.052 \\ 50 & 1.034 \\ 5 & 1.029 \\ 1 & 1.022 \\ 5 & 1.005 \\ 50 & 0.9954 \\ 1 & 0.9928 \\ 30 & 0.9862 \\ 1 & 0.9817 \\ 15 & 0.9796 \\ 1 & 0.9730 \\ 3 & \end{array}$

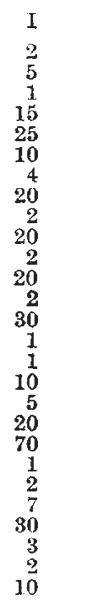

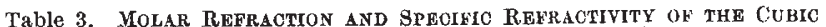
Table 3. MOLAR RIGH-PRESSUR POLYMORPHS OF $\mathrm{ZrP}_{2} \mathrm{O}_{7}$

\begin{tabular}{|c|c|c|c|c|}
\hline Polymorph & $\begin{array}{l}\text { Mean } \\
\text { refractive } \\
\text { Index }(n)\end{array}$ & $\begin{array}{c}\text { Density }(d) \\
\text { g/c.c. }\end{array}$ & $\begin{array}{l}\underset{\text { Molar }}{\text { refaction }} \\
\left(\frac{n^{2}-1}{n^{2}+2} \cdot \frac{M}{d}\right)\end{array}$ & $\begin{array}{c}\text { Specific } \\
\text { refractivity } \\
\qquad\left(\frac{n-1}{d}\right)\end{array}$ \\
\hline Cubic & $1 \cdot 661$ & $3 \cdot 13$ & $31 \cdot 31$ & 0.211 \\
\hline High-pressure & $1.761 *$ & $3 \cdot 62$ & $30-17$ & 0.210 \\
\hline
\end{tabular}

$$
\sqrt[3]{\omega^{2} \varepsilon}
$$

$M=$ inolecular weight

with $\varepsilon=1.773, \omega=1.755$, both \pm 0.003 , and $\varepsilon-\omega=0.018$. The crystallites have an average size of about $20 \mu$, but a few crystals as large as $40 \mu$ were observed. The crystallites are anhedral so there is no direct indication whether this phase is tetragonal or hexagonal. X-ray powder diffraction data are given in Table 2. The density of the new phase is $3 \cdot 62 \pm 0.02$ as determined by float-sink methods using Clerici's solution. The high-pressure phase, therefore, is 15.7 per cent denser than the cubic form, which has a density of $3 \cdot 13$, calculated from its lattice parameter.

The high-pressure phase inverts to the cubic form within $3 \mathrm{~h}$ when heated at ambient pressure in air at $1,000^{\circ} \mathrm{C}$.

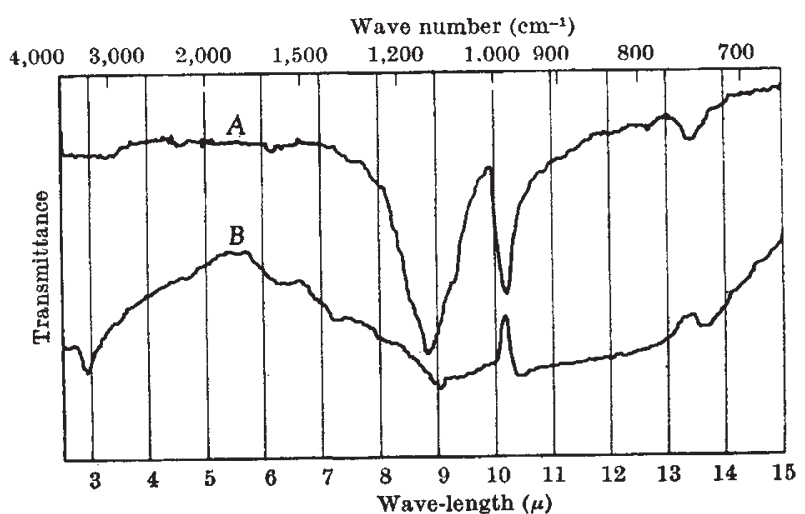

Fig. 1. Infra-red absorption spectra of the cubic and high-pressure polymorphs of $\mathrm{ZrP}_{8} \mathrm{O}_{7}$. Spectra obtained with Perkin-Elmer Infracord recording spectrophotometer using the $\mathbf{K B r}$ disk technique. $A$, cubic form preheated at $700^{\circ} \mathrm{C}$; $B$, high-pressure form 